\title{
Observatorio de Equidad de Género en Salud - Chile: Una mirada a las políticas públicas
}

\author{
PAMELA EGUIGUREN ${ }^{(1)}$, MARÍA EUGENIA CALVIN ${ }^{(2)}$, XIMENA DÍAZ $^{(3)}$, MARCELA FERRER $^{(4)}$, MARGARITA \\ IGLESIAS $^{(5)}$ y JOSÉ OLAVARRÍA ${ }^{(6)}$
}

En agosto de este año se dio a conocer el Informe del Observatorio de Equidad de Género en Salud 2007-2008. Este Observatorio, que ya tiene varios años de funcionamiento, se inicia a partir del proyecto OPS de Equidad Género y Reforma entre los años 2003-2006, con la visión y liderazgo de la Dra. María Isabel Matamala en su calidad de asesora en OPS. Es concebido desde entonces como una herramienta de vigilancia ciudadana en materia de reforma y políticas públicas de salud. Participaron en el proceso de construcción del Observatorio una serie de instituciones académicas y de la sociedad civil que hoy, sin el proyecto OPS que le dio origen, siguen dando vida a este espacio de observación y monitoreo, ciudadano. La información acerca del Observatorio, su estructura y los datos en los que basa su vigilancia son accesibles a través de su página web www.observatoriogenerosalud.cl, donde también es posible acceder a sus publicaciones.

El Observatorio ha definido 112 indicadores de equidad de género que se encuentran agrupados en ocho grandes campos de observación: Contexto social y demográfico, Situación general de salud, Atención de salud, Calidad de atención, Financiamiento y presupuesto público, Recursos Humanos de la salud, Participación en salud y Componentes específicos de la situación de salud, entre los que se han relevado indicadores de Salud sexual y reproductiva, Violencia de género, Salud mental, Salud y trabajo.

Para el seguimiento y evaluación de los indicadores que vigila, el Observatorio actualiza periódicamente los indicadores, realiza el análisis de su estado de avance en función de metas o criterios que significan una mayor equidad de género. En ese sentido se valoran avances cuando el valor se acerca a una situación más equitativa, estancamiento cuando no se registra modificación y retroceso cuando la situación de inequidad crece o, si se trata de políticas, cuando ha transcurrido tiempo y la situación no se modifica.

En el informe que da origen a esta entrega, se incluyó la visión de las organizaciones de la sociedad civil que participan en el "Foro Civil del Observatorio" y que enriquecieron con su mirada el análisis de los indicadores.

\section{Informe 2007-2008*}

En el campo de los indicadores sociodemográficos, se señalan aspectos sociales de carácter estructural y estadísticas de morbilidad y mortalidad, resaltando las desigualdades de género que estas cifras expresan. En términos de perfil demográfico las cosas no han cambiado mucho respecto del informe anterior. Nuestra población envejece y la proporción de mujeres respecto de los hombres mayores de 80 años continúa siendo

(1) Escuela de Salud Pública. Facultad de Medicina. Universidad de Chile. Independencia 939. Santiago. Chile. peguiguren@med.uchile.cl.

(2) Educación Popular en Salud, EPES. Chile.

(3) Proyecto Araucaria, Centro de Estudios de la Mujer, CEM. Chile.

(4) Departamento de Sociología. Facultad de Ciencias Sociales. Universidad de Chile. Chile.

(5) Centro de Estudios de Género y Cultura de América Latina. Universidad de Chile. Chile.

(6) Centro de Estudios de la Mujer. CEDEM. Chile.

$\left(^{*}\right) \quad$ A pesar de ser este el período de observación existen indicadores cuya última actualización disponible corresponde al año 2006. 


\begin{tabular}{|c|c|c|c|}
\hline & 2001 & 2006 & ESTADO \\
\hline $\begin{array}{l}\text { Porcentaje } \\
\text { de población } \\
\text { de } 75 \text { años y } \\
\text { más autova- } \\
\text { lentes por } \\
\text { sexo }\end{array}$ & $\begin{array}{l}\mathbf{5 4 . 2} \text { mujeres } \\
\text { de cada } 100 \\
\text { de } 75 \text { años y } \\
\text { más son auto- } \\
\text { valentes } 78.2 \\
\text { hombres de } \\
\text { cada } 100 \text { de } \\
75 \text { años y } \\
\text { más son auto- } \\
\text { valentes }\end{array}$ & $\begin{array}{l}\mathbf{4 4 . 6} \text { mujeres } \\
\text { de cada } 100 \\
\text { de } 75 \text { años y } \\
\text { másdeclaran } \\
\text { no tener mu- } \\
\text { cha dificultad } \\
\text { para ver-leer, } \\
\text { oír, hablar, } \\
\text { desplazarse, } \\
\text { masticar, to- } \\
\text { mar/manipul } \\
\text { ar objetos y } \\
\text { controlar es- } \\
\text { fínteres. El } \\
\mathbf{5 1 . 7 \%} \text { de los } \\
\text { hombres de- } \\
\text { clara lo mis- } \\
\text { mo }\end{array}$ & $\begin{array}{l}\begin{array}{l}\text { Sin valor de } \\
\text { referencia }\end{array} \\
\text { Los valores } \\
\text { de cada año } \\
\text { no son com- } \\
\text { parables para } \\
\text { analizar su } \\
\text { tendencia }\end{array}$ \\
\hline
\end{tabular}

bastante mayor, con cerca de 180 mujeres por cada 100 hombres sobre esa edad. A la par de esa constatación está el hecho de que las mujeres que alcanzan edades avanzadas se encuentran con mayores restricciones de salud que los hombres en esas condiciones, lo cual se aprecia en lo que declaran mujeres y hombres mayores de 75 años en la Encuesta de calidad de vida y salud del año 2006.

No solo las restricciones de salud acompañan este período a las mujeres, su disponibilidad de recursos económicos en esas edades es menor por sus dificultades de participación sostenida en el empleo remunerado. Las diferencias salariales en desmedro de las mujeres son reconocidas y también sus trayectorias laborales con más interrupciones debidas a los roles de género asumidos, llegando así a jubilar con pensiones más bajas.

El informe retoma planteamientos hechos con anterioridad respecto de la necesidad de que las políticas públicas reconozcan y aborden esta mayor vulnerabilidad en su formulación, tanto en salud como en el ámbito de políticas intersectoriales. En ese contexto el informe 2007-2008 valora como un avance la existencia de la "pensión básica solidaria" de la ley de reforma previsional a la que pueden optar mujeres sin antecedentes de cotización en el sistema de fondos de pensiones, y los beneficios de bonificación por hijo/as para mujeres con antecedentes de cotización. Estas medidas no solo tienen el impacto económico directo, sino también, lo que resulta fundamental para los cambios en la estructura de producción de inequidad, en el cambio cultural que otorga reconocimiento al trabajo doméstico y de cuidados no remunerado de las mujeres y su aporte a la sociedad.

En esa misma línea se valora la aplicación de la Encuesta de uso del tiempo en Gran Santiago, realizada por el INE y Minsal, que ha aportado datos poblacionales sobre las diferencias en el uso del tiempo entre hombres y mujeres, haciendo visible el trabajo de las mujeres en el hogar, donde quienes trabajan remuneradamente dedican además en promedio 2,9 horas diarias a estos trabajos; esto frente a menos de una hora diaria $(0,8)$ que dedican los hombres. Se demuestra así una carga global de trabajo diario mayor en las mujeres.

En cuanto a desigualdades en los resultados de salud basadas en la construcción social de ser hombres y mujeres en nuestra sociedad el Observatorio destaca entre sus indicadores, la brecha de carga de enfermedad en el país. El estudio realizado por el Minsal sobre las muertes ocurridas en 2004, muestra que las mujeres pierden más años de vida saludable por discapacidad que los hombres. La principal causa para esta pérdida en mujeres entre los 20 y 44 años es la depresión. Por su parte, los hombres pierden más años de vida que las mujeres por

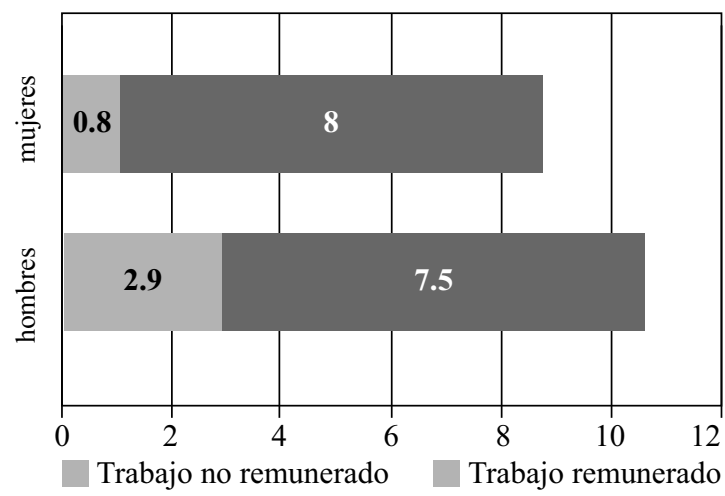

Fuente: Encuesta experimental sobre uso del tiempo en el gran Santiago. INE-MINSAL 2008. 


\begin{tabular}{|c|c|c|c|c|c|}
\hline & 2003 & 2004 & 2005 & 2006 & ESTADO \\
\hline $\begin{array}{l}\text { Sobremortalidad } \\
\text { masculina por } \\
\text { accidentes, } \\
\text { agresiones } \\
\text { autoinflingidas y } \\
\text { de otros, en } \\
\text { relación a la } \\
\text { mortalidad de las } \\
\text { mujeres por las } \\
\text { mismas causas, en } \\
\text { el grupo deedades } \\
\text { de } 15 \text { a } 34 \text { años }\end{array}$ & $\begin{array}{l}\mathbf{6 4 . 2} \\
\text { menos mujeres que } \\
\text { hombres por cada } \\
100 \text { mil murieron } \\
\text { por causas externas, } \\
\text { entre los } 15 \text { y } 34 \\
\text { años } \\
\text { Mujeres: } 11 \\
\text { Hombres: } 75.2\end{array}$ & $\begin{array}{l}\mathbf{6 4 . 3} \\
\text { menos mujeres que } \\
\text { hombres por cada } \\
100 \text { mil murieron } \\
\text { por causas externas, } \\
\text { entre los } 15 \text { y } 34 \\
\text { años } \\
\text { Mujeres: } 13.2 \\
\text { Hombres: } 77.5\end{array}$ & $\begin{array}{l}\mathbf{6 4 . 7} \\
\text { menos mujeres que } \\
\text { hombres po cada } \\
100 \text { mil murieron } \\
\text { por causas externas, } \\
\text { entre los } 15 \text { y } 34 \\
\text { años } \\
\text { Mujeres: } 13.2 \\
\text { Hombres: } 77.5\end{array}$ & $\begin{array}{l}\mathbf{6 2 . 5} \\
\text { menos mujeres que } \\
\text { hombres por cada } \\
100 \text { mil murieron } \\
\text { por causas externas, } \\
\text { entre } 15 \text { y } 34 \text { años } \\
\text { Mujeres: } 13.2 \\
\text { Hombres: } 77.5\end{array}$ & $\begin{array}{l}\text { Retroceso } \\
\text { La brecha } \\
\text { disminuye pero a } \\
\text { pesar de una ligera } \\
\text { disminución de la } \\
\text { tasa de mortalidad } \\
\text { en hombres, la tasa } \\
\text { en mujeres } \\
\text { aumentó. }\end{array}$ \\
\hline
\end{tabular}

muerte prematura. Esto se ve reflejado en otro indicador, que da cuenta de la sobre mortalidad masculina, especialmente en jóvenes, por accidentes y lesiones autoinflingidas, donde los varones entre los 15 y 34 años registran 76.5 muertes x 100.000 frente a $14 \times 100.000$ en mujeres en ese rango de edad.

Un grave problema de salud pública que afecta a las mujeres es la violencia de género. Ha aumentado la tasa de denuncias de violencia intrafamiliar, también la tasa de denuncia de abuso sexual y violaciones en niños y niñas menores de 14 años, así como en mujeres entre los 15 y 19 años. Esto habla de la magnitud del problema y se considera un avance por las dificultades históricas para su reconocimiento y denuncia. Se considera un dato asociado a violencia sexual el registro de 954 nacimientos en menores de 15 años en 2006.

Preocupa la alta frecuencia de femicidios (59 en 2008); que son los que llegamos a conocer, pues no existen aún registros oficiales de los asesinatos de mujeres por parte de sus parejas, lo cual el Observatorio plantea en forma de demanda. En este contexto ha sido importante la promulgación de una "Política de Salud en Violencia de Género" de Minsal. De ella el Observatorio destaca el enfoque de género, el reconocimiento de un problema de salud pública y la intersectorialidad; y valora que en su proceso de construcción hubo activa participación de la sociedad civil.

Como retroceso en este campo se destaca que el carácter integral e intersectorial de las acciones que se necesitan y que se proponen, no cuentan con el suficiente respaldo presupuestario, normativo y procedimental para garantizar su cumplimiento en calidad y oportunidad. En este caso la sociedad civil percibe la insuficiencia de las acciones en violencia de género, planteando que las iniciativas existentes muchas veces se muestran como una solución cuando están lejos de serlo, y observan una fragmentación de la respuesta, considerado esto un retroceso respecto de la respuesta integral que se requiere.

En materia de salud y trabajo, el Observatorio destaca que se haya iniciado un proceso de revisión y rediseño del programa de salud de los(as) trabajadores(as) por parte de la Subsecretaría de Salud Pública donde el énfasis está en la perspectiva de equidad y los determinantes sociales de la salud. Además se ha puesto en marcha un Sistema Nacional de Información en salud ocupacional, que centraliza y homologa los sistemas de registro de enfermedades y accidentes del trabajo, de modo de contar con buena información para el monitoreo, contribuyendo al avance en los temas de reconocimiento y notificación de enfermedades del trabajo. En contrapartida a algunos avances registrados en materia legislativa en este campo, donde a partir de marzo de 2006 se reconocen nuevas enfermedades, no ha habido nuevas iniciativas tendientes a actualizar el listado, especialmente en lo referido a trastornos emocionales. El Observatorio considera una omisión grave que la última actualización en 2006 no incluyera el "acoso sexual" como factor de riesgo para 
la salud mental en el trabajo, y un retroceso el hecho de que no existan iniciativas en proceso para su inclusión. El año 2006 hubo en el país 321 denuncias por acoso sexual, un $31 \%$ más que en el año anterior. En la mitad de los casos el acosador fue un superior jerárquico, en el $42 \%$ fue un compañero. Este es un problema que afecta mayoritariamente a las mujeres. Durante 2006 hubo sólo siete denuncias de hombres, correspondientes al 2\% del total, y solo un caso se refería al acoso de una mujer en contra de un hombre.

En cuanto a salud sexual y reproductiva, se hace referencia a la tasa de mortalidad materna, cifra que se aproxima a las 20 muertes por 100.000 nacidos vivos. Desde allí se destaca un $15 \%$ de muertes por aborto, donde en los últimos años han ocurrido muertes por causas que son indicación de aborto terapéutico, como embarazo ectópico y mola hidatiforme. Legítimamente se cuestiona la eliminación del aborto terapéutico en el código sanitario y su posible influencia en la falta de acceso oportuno de las mujeres a una interrupción en esta situación. Se plantea además que dadas las restricciones y actual penalización del aborto, la disminución de la mortalidad materna a causa de complicaciones de aborto ha sido y continúa siendo financiada por las mujeres, que asumen los costos del acceso a abortos más seguros.
El Observatorio también reitera su preocupación frente al alza sostenida en la proporción de cesáreas realizadas en el país, muy por sobre las recomendaciones del 15\% establecido por OMS. Preocupa que siendo este un ámbito de decisión médica esté reflejando otras motivaciones para su indicación, sometiendo a las mujeres a un riesgo injustificado. Es necesario que ellas tengan claridad respecto de las ventajas que tiene el parto normal frente a una intervención quirúrgica como es la cesárea y participen más activamente en esta decisión.

Como positivo destaca el aumento sostenido de los partos acompañados en el Sistema Público de Salud que alcanza a un $60 \%$ y en ese orden también el aumento de la atención intercultural del parto, donde se recogen experiencias que avanzan sostenidamente. Se destaca también la publicación del "Manual de Atención Personalizada en el Proceso Reproductivo", aunque es lamentable que no incluya la atención humanizada del aborto inseguro provocado. En un marco de penalización y de tradición conservadora en este tema, las mujeres que llegan en proceso de aborto a los hospitales deben ser protegidas de tratos vejatorios. Aunque se escapa del período de observación, en el lanzamiento del Informe se menciona el envío a los Servicios de Salud de un Ordinario (A15/1675) por parte

Tasas de Mortalidad Materna 2001-2006

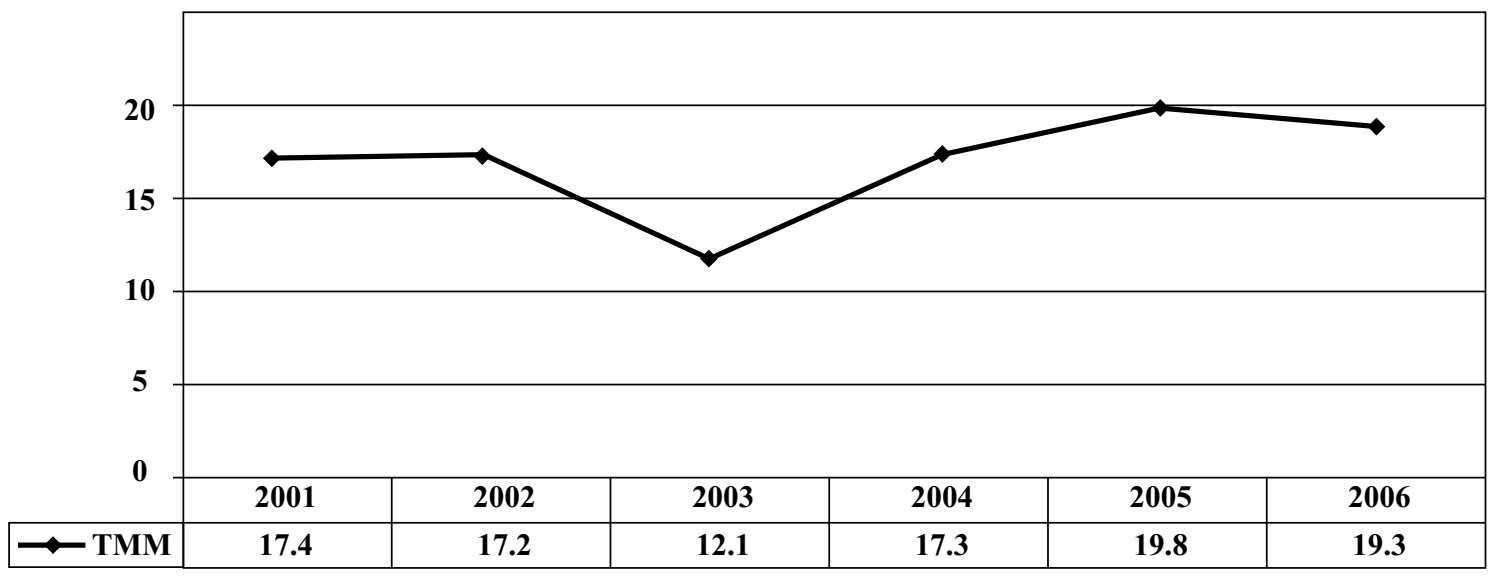

Fuente: Minsal. Departamento de estadísticas de información en salud. 


\begin{tabular}{|c|c|c|c|c|c|c|c|}
\hline \multirow[b]{2}{*}{$\begin{array}{l}\text { Tasa de denuncias } \\
\text { de abusos y } \\
\text { violaciones a } \\
\text { adolescentes } \\
\text { mujeres (14 a } 19 \\
\text { años) }\end{array}$} & 2005 & & 2006 & \multicolumn{2}{|c|}{2007} & 2008 & \multirow[b]{2}{*}{$\begin{array}{l}\text { Avance en } \\
\text { la denuncia }\end{array}$} \\
\hline & $\begin{array}{l}185.92 \\
\text { Denuncias x } 100 \\
\text { mil mujeres entre } \\
\text { los } 15 \text { y } 19 \text { años }\end{array}$ & \multicolumn{2}{|c|}{$\begin{array}{l}210.1 \\
\text { Denuncias x } 100 \\
\text { mil mujeres entre } \\
15 \text { y } 19 \text { años }\end{array}$} & \multicolumn{2}{|c|}{$\begin{array}{l}257.43 \\
\text { Denuncias x } 100 \\
\text { mil mujeres entre } \\
15 \text { y } 19 \text { años }\end{array}$} & $\begin{array}{l}285.05 \\
\text { Denuncias x } 100 \\
\text { mil mujeres entre } \\
15 \text { y } 19 \text { años }\end{array}$ & \\
\hline \multirow[b]{2}{*}{$\begin{array}{l}\text { Número de } \\
\text { nacimientos de } \\
\text { madres menores } \\
\text { de } 15 \text { años }\end{array}$} & \multicolumn{2}{|l|}{2004} & \multicolumn{2}{|c|}{2005} & \multicolumn{2}{|r|}{2006} & \\
\hline & \multicolumn{2}{|c|}{$\begin{array}{l}906 \\
\text { nacimientos de madres } \\
\text { menores de } 15 \text { años }\end{array}$} & \multicolumn{2}{|c|}{$\begin{array}{l}935 \\
\text { nacimientos de madres } \\
\text { menores de } 15 \text { años }\end{array}$} & \multicolumn{2}{|c|}{$\begin{array}{l}954 \\
\text { nacimientos de madres } \\
\text { menores de } 15 \text { años }\end{array}$} & Retroceso \\
\hline \multirow[b]{2}{*}{$\begin{array}{l}\text { Tasa de denuncias } \\
\text { de abusos y } \\
\text { violaciones a niñas } \\
\text { y a niños menores } \\
\text { de } 14 \text { años, por } \\
100.000\end{array}$} & 2005 & & 2006 & \multicolumn{2}{|c|}{2007} & 2008 & \\
\hline & $\begin{array}{l}\text { Niñas: } \\
67.7 \text { denuncias } \\
\text { de abusos y } \\
\text { violaciones por } \\
100 \text { mil } \\
\text { Niños: } \\
20.6 \text { denuncias } \\
\text { de abusos y } \\
\text { violaciones por } \\
100 \text { mil }\end{array}$ & \multicolumn{2}{|c|}{$\begin{array}{l}\text { Niñas: } \\
88.8 \text { denuncias } \\
\text { de abusos y } \\
\text { violaciones por } \\
100 \text { mil }\end{array}$} & \multicolumn{2}{|c|}{$\begin{array}{l}\text { Niñas: } \\
111.09 \text { denuncias } \\
\text { de abusos y } \\
\text { violaciones por } \\
100 \text { mil }\end{array}$} & $\begin{array}{l}\text { Niñas: } \\
137.87 \text { denuncias } \\
\text { de abusos y } \\
\text { violaciones por } \\
100 \text { mil } \\
\text { Niños: } \\
40.69 \text { denuncias } \\
\text { de abusos y } \\
\text { violaciones por } \\
100 \text { mil }\end{array}$ & $\begin{array}{l}\text { Avance en } \\
\text { la denuncia }\end{array}$ \\
\hline
\end{tabular}

del Ministro, que avanza una política de protección de las mujeres en esta situación, eliminando acciones relacionadas a la denuncia por parte del personal de salud.

El acceso a la anticoncepción de emergencia sigue como materia pendiente. El Observatorio al lanzar su informe 2007-2008 recoge la historia desde la introducción de las Normas Nacionales de Regulación de la Fertilidad, pasando por el fallo del Tribunal Constitucional, el fallo de Contraloría y se refiere a los primeros avances en el parlamento del nuevo proyecto de ley presentado para avanzar en las garantías de su libre acceso. El Observatorio valora la iniciativa del ejecutivo y espera poder dar cuenta de avances en el siguiente período.

En cuanto al seguimiento de políticas relacionadas a la equidad en el acceso, calidad y financiamiento de la salud se sigue la tramitación de proyectos de Ley. Varios de ellos no muestran avances: el Proyecto sobre derechos y deberes de las personas en salud, (donde hay temas tan sensibles y necesarios de regular como el consentimiento informado y los derechos en materia de salud a partir de los 14 años) y la ley que establece el derecho, en condiciones de desempleo a ser beneficiario/a de FONASA por ser cónyuge o conviviente de un o una cotizante. También se ha resaltado como retroceso lo que ha sucedido con la presentación sin avances del Proyecto de Ley marco sobre derechos sexuales y reproductivos y con las diversas tentativas de proyectos para la reposición del aborto terapéutico, donde urge su despenalización.

En los temas de Participación se valora contar con el Consejo Consultivo de Género y Salud de las Mujeres del Ministerio de Salud, espacio que propicia la participación de las organizaciones de la sociedad civil para hacer aportes en el trabajo de la institucionalidad pública, aunque también se plantean en este informe críticas al proceso.

En su informe el Observatorio hizo presente 
que la Presidenta Michelle Bachelet ha abordado deudas históricas con la salud de las mujeres y a pesar de que aún hay importantes temas pendientes, reconoce el aporte durante su mandato. Los retrocesos y estancamientos detectados plantean desafíos para la política pública que serán objeto de particular vigilancia y abogacía durante el próximo período de observación. Se esperan acciones desde el gobierno en sus distintas entidades y desde el ministerio de salud, orientadas hacia una mayor equidad de género.

Los datos presentados corresponden a la publicación Informe 2007-2008 del Observatorio de Equidad de Género en Salud. Santiago de Chile 2009.

Usted puede comentar éste y otros artículos publicados en la Revista Chilena de Salud Pública, enviando un correo electrónico a revistasp@med.uchile.cl 